\title{
Temperature dependent broadband impedance spectroscopy on poly-(p- phenylene-vinylene) light-emitting diodes
}

Cite as: Journal of Applied Physics 83, 5045 (1998); https://doi.org/10.1063/1.367321

Submitted: 02 February 1998 . Accepted: 11 February 1998 . Published Online: 28 April 1998

J. Scherbel, P. H. Nguyen, C. Paasch, W. Brütting, and M. Schwoerer

ARTICLES YOU MAY BE INTERESTED IN

Light-emitting diodes based on poly-p-phenylene-vinylene: II. Impedance spectroscopy Journal of Applied Physics 82, 1961 (1997); https://doi.org/10.1063/1.366004

Impedance spectroscopy as a probe for the degradation of organic light-emitting diodes Journal of Applied Physics 107, 054501 (2010); https://doi.org/10.1063/1.3294642

Negative capacitance in organic light-emitting diodes

Applied Physics Letters 86, 073509 (2005); https://doi.org/10.1063/1.1865346
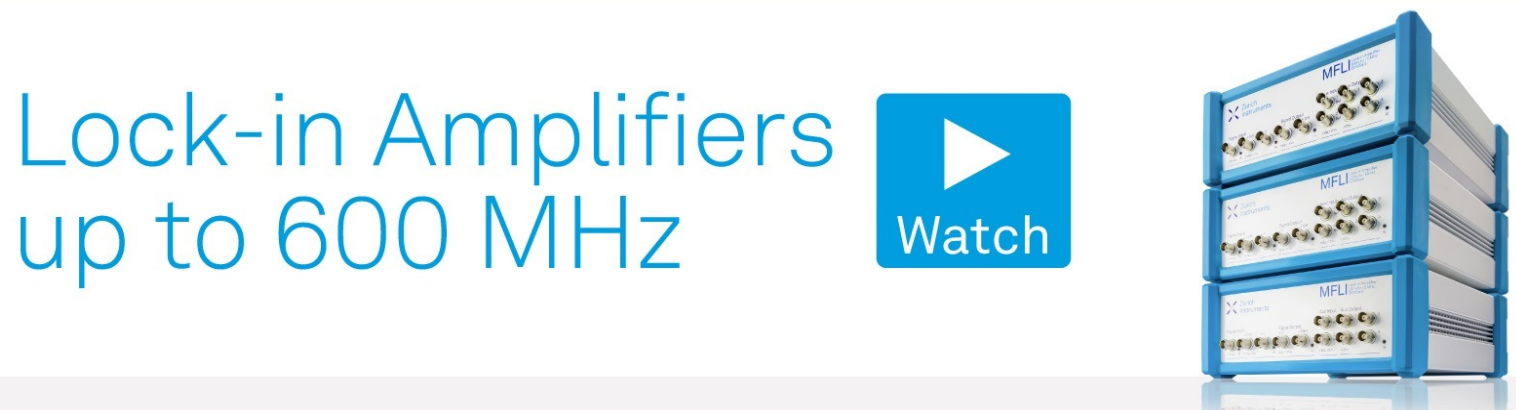


\title{
Temperature dependent broadband impedance spectroscopy on poly-(p-phenylene-vinylene) light-emitting diodes
}

\author{
J. Scherbel \\ Lehrstuhl für Experimentalphysik II, Universität Bayreuth, 95440 Bayreuth, Germany \\ P. H. Nguyen \\ Lehrstuhl für Experimentalphysik II, Universität Bayreuth, 95440 Bayreuth, Germany and Institut für \\ Festkörper- und Werkstofforschung Dresden, 01171 Dresden, Germany
}

G. Paasch

Institut für Festkörper- und Werkstofforschung Dresden, 01171 Dresden, Germany and Institut für Festkörperelektronik, Technische Universität Ilmenau, 98684 Ilmenau, Germany

W. Brütting ${ }^{\text {a) }}$ and M. Schwoerer

Lehrstuhl für Experimentalphysik II, Universität Bayreuth, 95440 Bayreuth, Germany

(Received 2 February 1998; accepted for publication 11 February 1998)

\begin{abstract}
Using temperature dependent impedance spectroscopy in a broad frequency range $\left(10^{-1}-10^{7} \mathrm{~Hz}\right)$, we have found that the ac behavior of indium-tin oxide (ITO)/poly-(p-phenylene-vinylene) (PPV)/aluminum light-emitting diodes shows several features which cannot be described by the usual simple double RC circuit representing a depleted junction region and an undepleted bulk. Instead, our measurements in combination with a theoretical modeling suggest that the PPV bulk is composed of a highly doped region at the ITO interface and a region with lower doping at a higher distance to the ITO. Moreover, the boundary between these two regions is not sharp but there is a gradual change in dopant concentration. The large frequency range allowed us to identify two distinct processes corresponding to the PPV bulk and a third one to the junction. The bulk relaxation frequencies correspond to the characteristic dielectric relaxation frequencies of charge carriers in the high and low conducting sublayers and are proportional to the respective conductivities. The magnitude and activation energy of the relaxation time correlates well with results obtained from temperature dependent DC conductivity measurements. For ITO substrates we obtain activation energies of $0.4 \mathrm{eV}$ and room temperature conductivity of about $10^{-7}$ and $10^{-9} \mathrm{~S} / \mathrm{cm}$ for the high and low conducting sublayers, respectively. On gold substrates only one bulk process and no junction process with an activation energy of about $0.6 \mathrm{eV}$ and a corresponding conductivity of $3 \times 10^{-11} \mathrm{~S} / \mathrm{cm}$ at room temperature is observed. The Schottky junction has been studied by temperature dependent capacitance-voltage spectroscopy at a low frequency of $0.16 \mathrm{~Hz}$. The obtained acceptor dopant concentration from $1 / C^{2}$ plots varies from $1.4 \times 10^{17}$ at room temperature to $6.9 \times 10^{16} \mathrm{~cm}^{-3}$ at $200 \mathrm{~K}$. Assuming a density of states between $5 \times 10^{20}$ and $5 \times 10^{21} \mathrm{~cm}^{-3}$ for the valence band the temperature dependent acceptor dopant density can be described with an acceptor ionization energy between 0.16 and $0.2 \mathrm{eV}$. (C) 1998 American Institute of Physics. [S0021-8979(98)03810-9]
\end{abstract}

\section{INTRODUCTION}

In the last ten years the semiconducting properties of conjugated polymers have been intensively studied because of their potential applications in electronics. Field effect transistors and Schottky diodes have been fabricated from a variety of materials. ${ }^{1-3}$ More recently light-emitting diodes (LEDs) based on conjugated polymers have attracted considerable interest for large area displays. ${ }^{4-8}$ In all of these applications the transport mechanisms in the polymer layer and the nature of the contacts between the electrodes and the polymer are of great importance.

For devices based on poly-(p-phenylene-vinylene) (PPV) and its derivatives both injection and bulk dominated

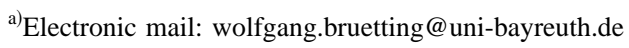

mechanisms have been proposed as the relevant processes for the current-voltage behavior. Parker et al. were able to fit the current-voltage characteristics of indium-tin oxide (ITO)/poly-[2-methoxy-5-(2'-ethyl-hexyloxy)-1,4-phenylenevinylene] (MEH-PPV)/metal devices using FowlerNordheim tunneling of holes. ${ }^{9}$ The obtained barrier heights correspond to the difference between the work function of ITO and the ionization potential of MEH-PPV. Similar behavior could also be observed for PPV devices at higher electric fields. ${ }^{10}$ The application of tunneling theory for charge carrier injection assumes an insulating material with rigid energy bands forming a neutral contact with the metal electrodes. Band bending effects, e.g., due to image force barrier lowering (Schottky effect), do only occur within a small distance to the interface. 
It has been shown recently by the Sheffield group ${ }^{11}$ that Fowler-Nordheim tunneling cannot satisfactory describe their data on thin $(d \approx 100 \mathrm{~nm})$ ITO/PPV/Al devices. Although reasonable values of the hole barrier $(0.3 \mathrm{eV})$ were obtained in the high field regime, the theory cannot account for the observed temperature dependence, the thickness dependence and the magnitude of the current at a given bias. Instead they could fit their device characteristics very well by space-charge limited conduction with an exponential distribution of traps with a characteristic energy of $0.15 \mathrm{eV}$ and a trap density of $5 \times 10^{17} \mathrm{~cm}^{-3}$.

Previous work by our group on thicker $(d>200 \mathrm{~nm})$ ITO/PPV/Al devices has shown that a Schottky barrier with a depletion layer of width $50-150 \mathrm{~nm}$ is present at the $\mathrm{Al} /$ PPV interface. ${ }^{12,13}$ From capacitance-voltage measurements an acceptor dopant concentration in the order of $10^{17} \mathrm{~cm}^{-3}$ was determined. The occurrence of doping in PPV devices is not unexpected, since in the used precursor route to PPV the conversion of the deposited precursor polymer film to the conjugated PPV is performed at elevated temperatures directly on the electrode used for hole injection in LEDs. The precursor leaving groups (especially $\mathrm{HCl}$ ) can easily react with the ITO substrate forming $\mathrm{InCl}_{3}$, which in turn can diffuse into the PPV layer and oxidize (dope) the polymer. The chemical reaction at the ITO substrate has been proven by energy-dispersive $\mathrm{x}$-ray analysis ${ }^{14}$ and the subsequent diffusion by secondary ion mass spectrometry (SIMS). ${ }^{15} \mathrm{~A}$ comparison of different substrate materials has shown that the degree of doping resulting from such chemical reactions depends on the reactivity of the substrate material. ${ }^{16}$ While PPV devices on Au substrates did not show measurable doping, in devices on ITO an acceptor dopant concentration of some $10^{17} \mathrm{~cm}^{-3}$ was found. Thus we could identify the usage of ITO anodes as a major source for doping in PPV devices. This doping is responsible for the formation of a Schottky contact at the interface between PPV and metals like $\mathrm{Ca}$ or Al. However, already the SIMS measurements of ITO/PPV/Al structures show an inhomogeneous depth profile of the elements $\mathrm{In}$ and $\mathrm{Cl}$ in the PPV film with a gradual increase of the In concentration towards the ITO substrate. Thus a simple equivalent circuit composed of a bulk and a junction region used before for the modeling of $\mathrm{dc}$ and ac device characteristics ${ }^{12}$ may not be sufficient for a description of these devices.

It should be mentioned that impedance spectroscopic investigations on PPV LEDs are reported in the literature by several groups, however, with different results and interpretations. Measurements performed on ITO/PPV/Al devices with Cambridge PPV can be described by one single RC circuit corresponding to a fully depleted bulk. ${ }^{17}$ The occurrence of two semicircles in the complex impedance plane on some devices was ascribed to the presence of an interfacial oxide layer at the PPV/Al contact. Apart from our results, the presence of a Schottky contact with a depletion layer was also observed by Antoniadis et al. on ITO/PPV/Al structures with an acceptor dopant concentration of some $10^{16} \mathrm{~cm}^{-3} \cdot{ }^{18}$ Nguyen et al. could identify three different processes on $\mathrm{Cr} /$ $\mathrm{PPV} / \mathrm{Cr}$ devices, which they interpreted as a bulk process with an activation energy of $0.4 \mathrm{eV}$ and two interfacial processes. ${ }^{19}$ However, the comparison with these data is problematic since the usage of $\mathrm{Cr}$ substrates may lead to different chemical reactions during the conversion of the precursor.

From this brief survey of literature data it is obvious that nominally the same PPV prepared by the tetrahydrothiophene leaving group precursor route shows different device characteristics. This is probably due to slight differences in the precursor synthesis, which lead to different degree of doping in devices. The Sheffield group, e.g., has recently compared identically prepared devices from Cambridge PPV and our PPV. ${ }^{11}$ While they find no evidence for a depletion region in devices from the Cambridge PPV, our PPV devices showed clear evidence for a Schottky junction in accordance with our previous work. In spite of these quantitative differences in the dopant concentration and the resulting different device characteristics, there are certainly some general properties common to the different PPVs. As an example, the Sheffield group has obtained a trap depth of $0.15 \mathrm{eV}$ from fitting their temperature dependent currentvoltage curves to space-charge limited currents with an exponential distribution of traps. This value is in excellent agreement with our results from thermally stimulated currents. ${ }^{20}$ Thus we regard our PPV Schottky diodes as useful model devices to study basic transport properties of PPV in LEDs, although a Schottky diode is not very favorable for efficient light-emitting devices (since it is a majority carrier device). For such devices a variety of experimental techniques and theory to study doping, trap states and other relevant parameters for charge transport is known from inorganic semiconductors.

In this article a detailed study of the ac impedance of PPV Schottky diodes in a wide frequency range $\left(10^{-1}-10^{7}\right.$ $\mathrm{Hz})$ as a function of temperature and dc bias is presented. Our focus was especially on the investigation and identification of different processes occurring in this large frequency window. From their frequency and temperature dependence one can expect more information on the transport parameters of PPV and the equivalent circuit necessary to describe the device characteristics. Bias dependent measurements should give information about depletion layers and the corresponding acceptor dopant concentration. From the temperature dependence of this quantity the energetic depth of the acceptors should be determined.

\section{EXPERIMENT}

Impedance or dielectric spectroscopy is a powerful tool to study relaxation and loss processes in organic and inorganic materials, both in solution and in the solid state. ${ }^{21-25} \mathrm{It}$ does not only allow to study material parameters like the frequency dependent complex dielectric constant (or equivalent the complex conductivity), but also the influence of interfaces between two different materials. The application of bias allows additional measurement techniques like capacitance-voltage spectroscopy, which is a well established method to investigate depletion regions and the corresponding doping or trap concentrations in semiconducting materials. 
Impedance and capacitance-voltage measurements were performed using a Solartron Schlumberger SI1260 Impedance/Gain Phase Analyzer combined with a broadband dielectric converter (Novocontrol) as preamplifier. This setup allows impedance measurements up to $10^{14} \Omega$ with a $\tan \delta$ resolution of $10^{-4}$ at best. The oscillator amplitude ranged between 0.1 and $1 \mathrm{~V}$ and bias up to $10 \mathrm{~V}$ could be applied in both polarities for frequencies higher than $0.1 \mathrm{~Hz}$. Measurements were performed in a continuous flow liquid nitrogen cryostat (Oxford Instr. CF 1200) with helium as exchange gas in the sample space. Due to the time of more than $10 \mathrm{~min}$ required for a frequency sweep from $10^{7}$ to $0.1 \mathrm{~Hz}$, temperature stability of better than $0.1 \mathrm{~K}$ over long time had to be achieved with a temperature controller.

Devices consisted of ITO coated glass substrates (Flachglas, $20 \Omega / \square$ ) on which the tetrahydrothiophene leaving group precursor PPV (synthesized by our group as described in Refs. 26 and 27) was deposited by a precision doctor blade technique as several hundred $\mu \mathrm{m}$ thick liquid films. After drying and conversion ( 2 to 3 hours in a vacuum of $10^{-2}$ mbar at $170-190^{\circ} \mathrm{C}$ ) films of some hundred nm thickness were obtained. Subsequently an Al electrode was vacuum evaporated at $10^{-5}$ mbar in a thickness of at least $100 \mathrm{~nm}$. By using perpendicular stripes of ITO and $\mathrm{Al}$ (each $5 \mathrm{~mm}$ wide) an active device area of $0.25 \mathrm{~cm}^{2}$ was obtained. After metal evaporation the glass substrates were cut into $10 \times 10 \mathrm{~mm}^{2}$ pieces containing only one device in order to avoid cross talk between the pixels. Electrical contacts were made by pressing metal tips onto the Al and ITO stripes outside of the active area.

Several devices with thickness ranging from 400 to 1000 $\mathrm{nm}$ were investigated. Thicknesses of the polymer films were determined using a Dektak surface profilometer. Prior to the impedance measurements on all devices dc current-voltage characteristics were measured with a Keithley 236 sourcemeasure unit. On some devices and on a free-standing 20$\mu$ m-thick PPV film (sandwiched between two gold plates) also temperature dependent dc conductivity measurements were performed in the two-probe configuration with a Keithley 617 electrometer.

For the analysis of the data it is important that there are different but equivalent immittances. The quantities obtained directly in impedance spectroscopy are the real and imaginary parts of the frequency-dependent complex impedance

$$
\hat{Z}=\frac{d \hat{U}}{d \hat{I}}=Z^{\prime}+J Z^{\prime \prime}
$$

which is one of the four equivalent immittances. The other impedance-related immittances can be obtained from the impedance. First is the admittance:

$$
\hat{Y}=\frac{1}{\hat{Z}}=Y^{\prime}+J Y^{\prime \prime}
$$

Further, one has the dielectric function $\hat{\varepsilon}(\omega)$ defined by $\hat{Y}$ $=J \omega \varepsilon_{0} \hat{\varepsilon} \frac{A}{d}$, or

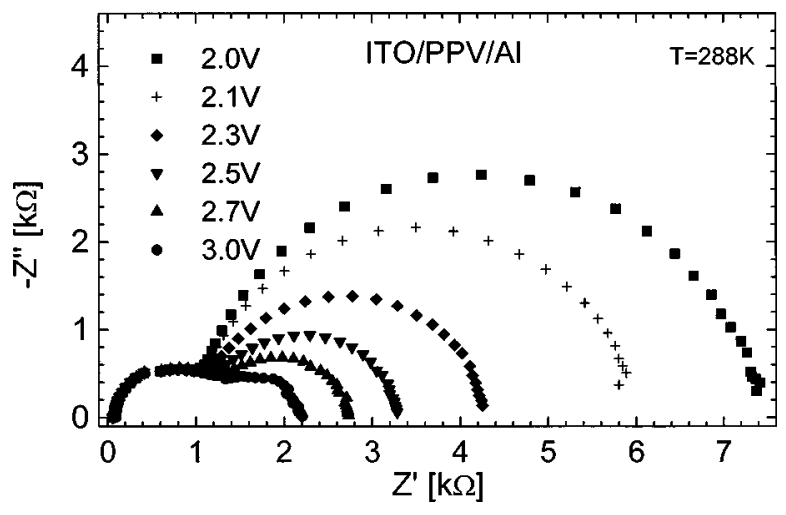

FIG. 1. Cole-Cole plot of the impedance of an ITO/PPV/Al sample $(d$ $=380 \mathrm{~nm}, A=25 \mathrm{~mm}^{2}$ ) at room temperature for various forward bias voltages. The frequency increases from right to left in the region between 100 $\mathrm{Hz}$ and $10 \mathrm{MHz}$. The left semicircles are assigned to the polymer bulk and the right semicircles to the depletion layer.

$$
\hat{\varepsilon}=\frac{1}{J \varepsilon_{0} \omega \hat{Z}_{\bar{d}}^{A}}=\varepsilon^{\prime}+J \varepsilon^{\prime \prime},
$$

where $\varepsilon_{0}$ is the vacuum permittivity, $d$ and $A$ are the thickness and the active area of the sample, respectively, and $\omega$ is the angular frequency. It has to be mentioned that contrary to the common meaning this dielectric function $\hat{\varepsilon}(\omega)$ is not simply a material property but it characterizes the whole system (described as a plate capacitor) including interfaces and also leads. Finally, the dielectric modulus is

$$
\hat{M}=\frac{1}{\hat{\varepsilon}}=J \varepsilon_{0} \omega \hat{Z} \frac{A}{d} .
$$

Though the four immittances are formally completely equivalent, depending on the system considered and the frequency regions of interest they are more or less suited for a direct graphical visualization of characteristic properties of the system. The reason lies in different possible asymptotic frequency dependences described by the different powers of the frequency $\omega$ as prefactor in the immittances. When a system behaves predominantly capacitively $\varepsilon^{\prime}(\omega)$ and $\varepsilon^{\prime \prime}(\omega)$ are particularly suited quantities and the usual ColeCole plot for the impedance, i.e., $-Z^{\prime \prime}=f\left(Z^{\prime}\right)$, gives only little insight, whereas the latter should be preferred when the resistive behavior dominates. In the case of the diodes considered here this is the case for forward bias, whereas the former is true for reverse bias.

\section{RESULTS AND DISCUSSION}

\section{A. Survey of the temperature and bias dependence in the complex impedance plane}

In this section we give an overview of the dielectric response of ITO/PPV/Al diodes. Therefore we discuss the impedance Cole-Cole plots for a given temperature and different positive applied voltages (forward), for a given forward bias and different temperatures as well as the ColeCole plot of the dielectric modulus for a given temperature for both forward and reverse bias.

Figure 1 shows an impedance Cole-Cole plot of an ITO/ 
PPV/Al sample $\left(A=25 \mathrm{~mm}^{2}, d=380 \mathrm{~nm}\right)$ under different forward bias in the frequency region between $100 \mathrm{~Hz}$ and 10 $\mathrm{MHz}$ at room temperature. In this diagram the implicit variable is the frequency $\omega$ which increases from right to left. Independently on bias voltage, all the curves in Fig. 1 display a small semicircle close to the origin corresponding to the high frequency region. With increasing forward bias the right semicircle (low frequencies) becomes smaller. Detailed investigations of the current-voltage and capacitancevoltage characteristics of ITO/PPV/metal LEDs ${ }^{12,13}$ have shown that the PPV/Al interface is a typical Schottky contact. In these Schottky diodes a depletion region is formed at the metal/polymer (Al/PPV) contact, which is accompanied by significant band bending and a high internal electrical field. In the measured forward bias region the Shockley equation $^{28}$ for the diode current is valid:

$$
I(U)=I_{0}\left\{\exp \left[\frac{q\left(U-I R_{B}\right)}{n k_{B} T}\right]-1\right\},
$$

where $n$ is the ideality factor and $R_{B}$ is the bulk resistance. For $\omega \rightarrow 0$ the real part of $\hat{Z}$ yields the total differential resistance of the sample

$$
Z^{\prime}(\omega \rightarrow 0)=R_{t}=\frac{d U}{d I}
$$

as obtained from the current-voltage characteristics. With Eq. (5) one obtains

$$
R_{t}=\frac{n}{I_{0}} \frac{k_{B} T}{q} \exp \left\{-\frac{q\left(U-R_{B} I\right)}{n k_{B} T}\right\}+R_{B} \equiv R_{j}+R_{B},
$$

where the bulk resistance $R_{B}$ is bias independent. Together with the corresponding capacity it leads to the small left semicircle in Fig. 1, which therefore is assigned to the bulk process. On the other hand, the resistance of the depletion layer $R_{j}=\left(n / I_{0}\right)\left(k_{B} T / q\right) \exp \left\{-q\left(U-R_{B} I\right) / n k_{B} T\right\}$ is strongly bias dependent. With increasing forward bias $R_{j}$ decreases exponentially, which is clearly seen in the right semicircles of Fig. 1. At forward bias of $U=3 \mathrm{~V}$ the depletion layer resistance amounts to only about $1 \mathrm{k} \Omega$. At further increase of the applied voltage the depletion layer and hence the corresponding resistance disappear. At the same time Fig. 1 shows that the bulk resistance $R_{B}$ cannot be neglected in the measured bias region. Therefore one cannot obtain quantitative information on the ideality factor of the diode from these experimental data in a simple manner.

Figure 2 shows the temperature dependence of the impedance of the same diode at forward bias of $U=2 \mathrm{~V}$. The left semicircle (i.e., the bulk resistance $R_{B}$ ) increases with decreasing temperature. This is a direct consequence of the thermally activated behavior of the conductivity of PPV. ${ }^{26,29,30}$ In contrast, the process in the Schottky contact does not show a monotonous behavior with temperature. At first, from $288 \mathrm{~K}$ to about $263 \mathrm{~K}$ the right semicircle (and thus the differential resistance of the Schottky contact) increases with decreasing temperature. However below $260 \mathrm{~K}$ the right semicircle decreases abruptly with decreasing temperature. One can understand the decrease of the differential resistance of the Schottky contact at lower temperatures with

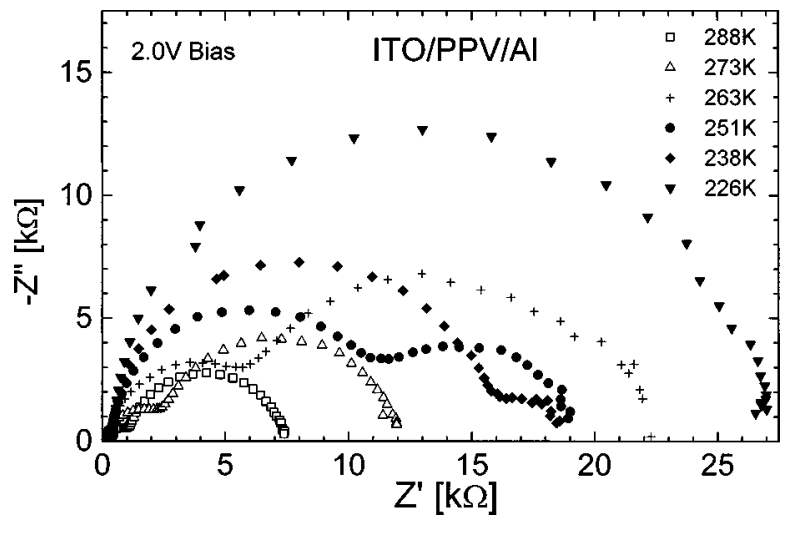

FIG. 2. Temperature dependent impedance spectra of the same sample of Fig. 1 at forwards bias $U=2 \mathrm{~V}$. The left semicircles assigned to the polymer bulk process increase with decreasing temperature. In contrast, the depletion layer process does not show a monotonous behavior with temperature.

the Shockley equation [or with Eq. (7)] qualitatively. At lower temperatures diffusion is the dominating transport mechanism in the Schottky contact since the carrier mobility is low. For this transport mechanism $I_{0}$ is only weakly temperature dependent and the exponential function [cf. Eq. (7)] determines the temperature dependence of $R_{j}$ essentially: with decreasing temperatures $R_{j}$ decreases exponentially. Thus the increase of $R_{j}$ with decreasing temperature down to about $260 \mathrm{~K}$ must be assigned to the presence of at least one other mechanism (besides diffusion) for the transport across the junction. The data indicate that at higher temperatures such transport mechanism becomes predominant. $I_{0}$ might then have a stronger temperature dependence in a given temperature region. This phenomenon requires further investigations.

In order to evaluate the experimental data it is common to use an equivalent circuit consisting of two parallel RC networks in a serial combination. In the equivalent circuit the depletion layer corresponds to a junction resistance $R_{j}$ and a junction capacitance $C_{j}$. Between the depletion layer and the positive electrode there exists a neutral bulk region represented in the equivalent circuit by a resistance $R_{B}$ and a capacitance $C_{B}$. The contact of PPV with the high-workfunction ITO is regarded as ohmic and has a negligible impedance in the ideal case. ${ }^{12,13}$ Depending on the thickness of the device and on the measured bias region a clear distinction of the individual resistances and capacitances in a ColeCole plot is not in any case possible as discussed in Ref. 12. If the system behaves at the applied bias voltage capacitively then the dielectric modulus or the dielectric function is better suited than the impedance itself to visualize the system properties in a Cole-Cole plot. In such a case the complex dielectric modulus (Fig. 3) shows essentially two semicircles (as Fig. 1 for the impedance) but the frequency as an implicit variable increases now from left to right. For high frequencies all the right-hand semicircles approach the same value corresponding to the geometrical capacitance $C_{\text {geo }}$ $=\varepsilon_{0} \varepsilon_{r} A / d$. Here $\varepsilon_{r}$ is the relative dielectric constant of the PPV which has to be clearly distinguished from the dielectric function $\hat{\varepsilon}$ defined in Eq. (3). At reserve bias voltage there exists only one semicircle because $C_{j}$ decreases and hence 


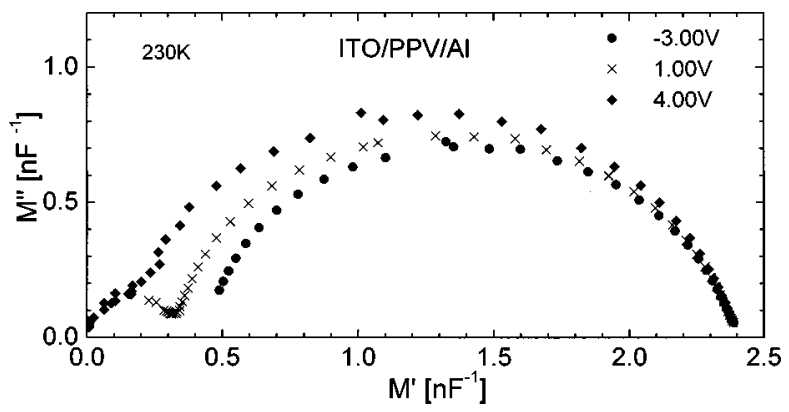

FIG. 3. Immittance spectrum of an ITO/PPV/Al diode $(d=1250 \mathrm{~nm}, A$ $=25 \mathrm{~mm}^{2}$ ) at different bias voltages in the complex dielectric modulus plane. The reciprocal of the right and left semicircle diameter determine the bulk and depletion layer capacitance, respectively.

the response of this circuit part is dominated by the resistance $R_{j}$ in the measured frequency region. Hence the second semicircle is suppressed. Thus for reverse bias the dielectric modulus plot seems to be rather structureless (only one semicircle). In the next section it will be demonstrated that contrary to the structureless dielectric modulus plot the frequency dependence of the dielectric function is extremely structured and allows for a detailed understanding of the processes in this region. It will be seen that an equivalent circuit consisting of two RC circuits as suggested from Fig. 1 and used before in Ref. 12 is not sufficient to describe the experimental data.

\section{B. Temperature dependence of the dielectric function at reserve bias}

At zero or reserve bias voltage the diodes behave predominantly capacitively. Therefore the dielectric function is the relevant immittance to present the experimental data graphically. Figure 4 shows the spectrum of the frequency dependent dielectric function of an ITO/PPV/Al diode with a thickness $d=700 \mathrm{~nm}$ and $A=25 \mathrm{~mm}^{2}$ at zero bias $U=0 \mathrm{~V}$. The imaginary part of the dielectric function $\varepsilon^{\prime \prime}(\omega)$ shows more than two maxima which have to be attributed to relaxation processes. (In contrast, the simple equivalent circuit mentioned above yields only two maxima.) The first of these processes lies at the frequency of about $2 \times 10^{6} \mathrm{~Hz}$ and is practically temperature independent. The second and third processes lie at room temperature between $2 \times 10^{4}-3 \times 10^{5}$ $\mathrm{Hz}$ and $10^{2}-4 \times 10^{2} \mathrm{~Hz}$, respectively. Both of these processes are temperature dependent, they move to lower frequencies with decreasing temperature and at low temperature they cannot be completely observed in the measured frequency window.

The real part of the dielectric function $\varepsilon^{\prime}$ decreases continuously with increasing frequency. Although one can see different relaxation processes also in $\varepsilon^{\prime}$ (i.e., hints of steps) they are much more smeared out than the maxima in $\varepsilon^{\prime \prime}$. At $0.1 \mathrm{~Hz} \varepsilon^{\prime}$ does not reach a saturation value in the measured temperature range. The drop of $\varepsilon^{\prime}$ or the maximum of $\varepsilon^{\prime \prime}$ above $10^{6} \mathrm{~Hz}$ are not connected with processes in the diode but they arise from the no-load capacitance and the lead resistance of the diode as will be seen below. For the explanation of the two other processes the simple equivalent circuit

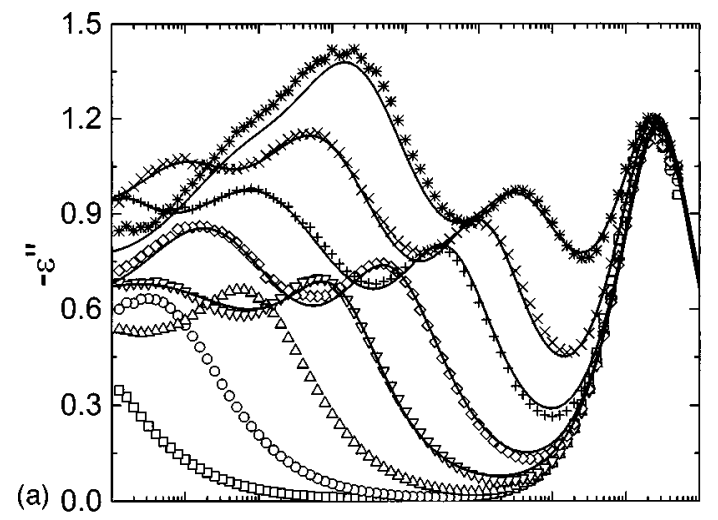

(a) 0.0

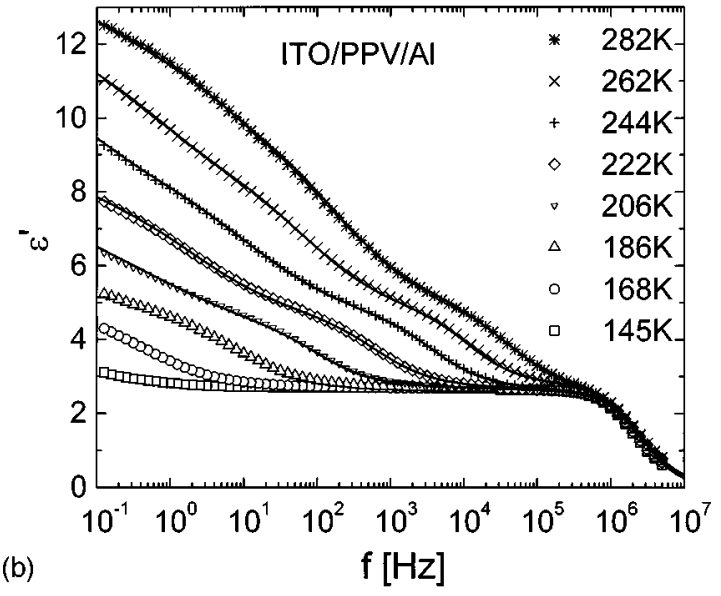

FIG. 4. Imaginary (a) and real part (b) of the dielectric function $\hat{\varepsilon}$ of an ITO/PPV/Al sample $\left(d=780 \mathrm{~nm}, A=25 \mathrm{~mm}^{2}\right)$ at different temperatures. There exist several clearly resolved maxima in $\varepsilon^{\prime \prime}$, which have to be attributed to relaxation processes. These processes are more smeared out in $\varepsilon^{\prime}$, which does not achieve saturation at lowest frequencies. The solid lines are fit curves.

mentioned above is not sufficient since it does not contain two thermally activated processes resulting in the observed shift with temperature. It will be shown now that it is the inhomogeneity of the PPV bulk which gives an explanation for these processes. Indeed, the PPV device fabrication can lead to a bulk inhomogeneity. Because PPV is insoluble, in our preparation route a prepolymer is used for film fabrication which has to be thermally converted to PPV. During the conversion aggressive leaving groups like $\mathrm{HCl}$ and tetrahydrothiophene are formed which can react with the ITO substrate to form compounds like $\mathrm{InCl}_{3}$ that can further oxidize (dope) the PPV. Since the oxidizing agents are mainly formed at the interface to ITO it is expected that a gradient in doping will exist in the PPV film with the highest value at the ITO. Such a gradient of $\mathrm{In}$ and $\mathrm{Cl}$ is indeed evident from the above mentioned SIMS measurements. Consequently, one cannot expect a constant conductivity profile throughout the whole PPV film.

Correspondingly, if one assumes the PPV layer to consist of several sublayers each of them with a width $d_{i}$, a constant resistance $R_{i}$ and a constant capacitance $C_{i}$, the impedance of such a sample with a lead resistance $R_{0}$ can be described by 


$$
\hat{Z}=R_{0}+\sum_{i} \frac{R_{i}}{1+J \omega C_{i} R_{i}}=R_{0}+\sum_{i} \frac{1}{\frac{1}{R_{i}}+J \omega C_{i}} .
$$

However, in a real sample the specific electrical resistivity will vary continuously and not step wise as assumed in Eq. (8). Now, substituting $C_{i}=\varepsilon_{0} \varepsilon_{r} A / d_{i}$ one can generalize Eq. (8) with $x=d_{i} / d$ simply to the case of a continuously varying resistivity [conductivity $\sigma(x)$ ] of the PPV as

$$
\hat{Z} \frac{A}{d}=R_{0} \frac{A}{d}+\int_{0}^{1} \frac{d x}{\sigma(x)+J \omega \varepsilon_{0} \varepsilon_{r}} .
$$

As before from Eqs. (9) with (3) the corresponding dielectric function can be obtained. We demonstrate now that the essential features seen in Fig. 4 are described by this model. Thereby we assume:

(i) The PPV layer can be divided roughly into three sublayers: the depletion layer $(j)$, a lower $(l)$, and a higher $(h)$ conductive bulk sublayer. Thus, the position dependence of the conductivity $\sigma(x)$ can be approximated by

$$
\begin{aligned}
\sigma(x)= & s_{j}+s_{l}\left\{\tanh \left[c_{l}\left(x-x_{l}\right)\right]+1\right\} \\
& +s_{h}\left\{\tanh \left[\left(c_{h}\left(x-x_{h}\right)\right]+1\right\},\right.
\end{aligned}
$$

where $s_{j}$ is the depletion layer conductivity and $2 s_{l}$ and $2 s_{h}$ are the conductivities of the lower and the higher conductive bulk sublayer, respectively. $c_{l}$ and $c_{h}$ are constants which determine the widths of the transition regions between the sublayers $\left(1 / c_{i}\right.$ is the width of the transition region in units of $d)$. As an example we assume $c_{l}=40$ and $c_{h}=15$. With these values the transition from the depletion to the lower conductive bulk sublayer occurs in a narrower region than that from the lower to the higher conductive sublayer. $x_{l}$ $=0.3$ and $x_{h}=0.65$ determine the sublayer widths relative to the total PPV thickness $\left(d=700 \mathrm{~nm}, A=25 \mathrm{~nm}^{2}\right): 1-x_{h}$ and $x_{h}-x_{l}$ are the widths of the higher and lower conductive bulk sublayers, respectively. Further we assume $s_{j}=10^{-15}$ $\mathrm{S} / \mathrm{cm}, s_{l}=2 \times 10^{-10} \mathrm{~S} / \mathrm{cm}, s_{h}=5 \times 10^{-8} \mathrm{~S} / \mathrm{cm}$ at $T=282 \mathrm{~K}$ and $R_{0}=112 \Omega$ (see below). As the dc conductivity of PPV shows a thermally activated behavior, ${ }^{26} s_{l}$ and $s_{h}$ are chosen to be temperature dependent with an activation energy of $E_{a}=0.4 \mathrm{eV}$ according to earlier measurements and to be confirmed below. For simplicity the other parameters are chosen independent of the temperature. Figure 5 shows this position dependence of the conductivity in the PPV layer for different temperatures and the corresponding equivalent circuit.

(ii) The relative dielectric constant $\varepsilon_{r}=2.4$ is assumed to be constant through the sample and to be temperature independent.

In Fig. 6 the calculated dielectric function for different temperatures based on these data is shown. In the model calculations maxima in $\varepsilon^{\prime \prime}$ are visible only if one of the resistances is much larger than the others (i.e., with an enormous specific resistance $\rho_{j}$ of the depletion layer). The first maximum from right in $\varepsilon^{\prime \prime}$ and the decrease of $\varepsilon^{\prime}$ in the frequency region above $10^{6} \mathrm{~Hz}$ are temperature independent. They are determined by the geometrical capacitance $C_{\text {geo }}$ and the lead resistance $R_{0}$ alone. Further model calculations
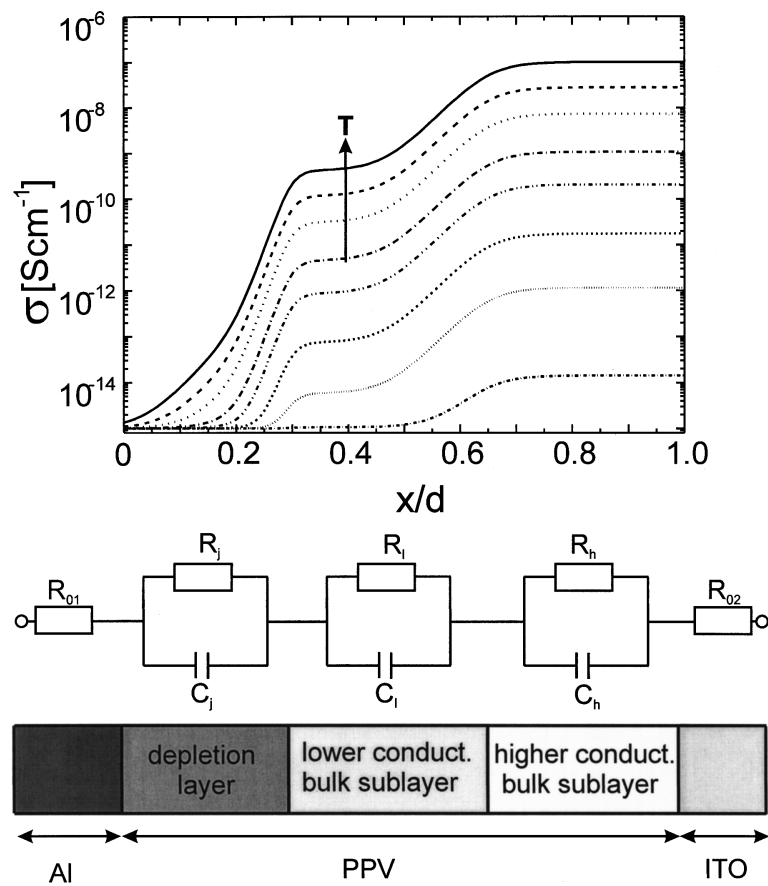

FIG. 5. Position dependence of the conductivity in the PPV layer used in the model calculation (Fig. 6) for different temperatures, the corresponding simplified equivalent circuit (for stepwise varying conductivity) and the connection to the layer sequence.
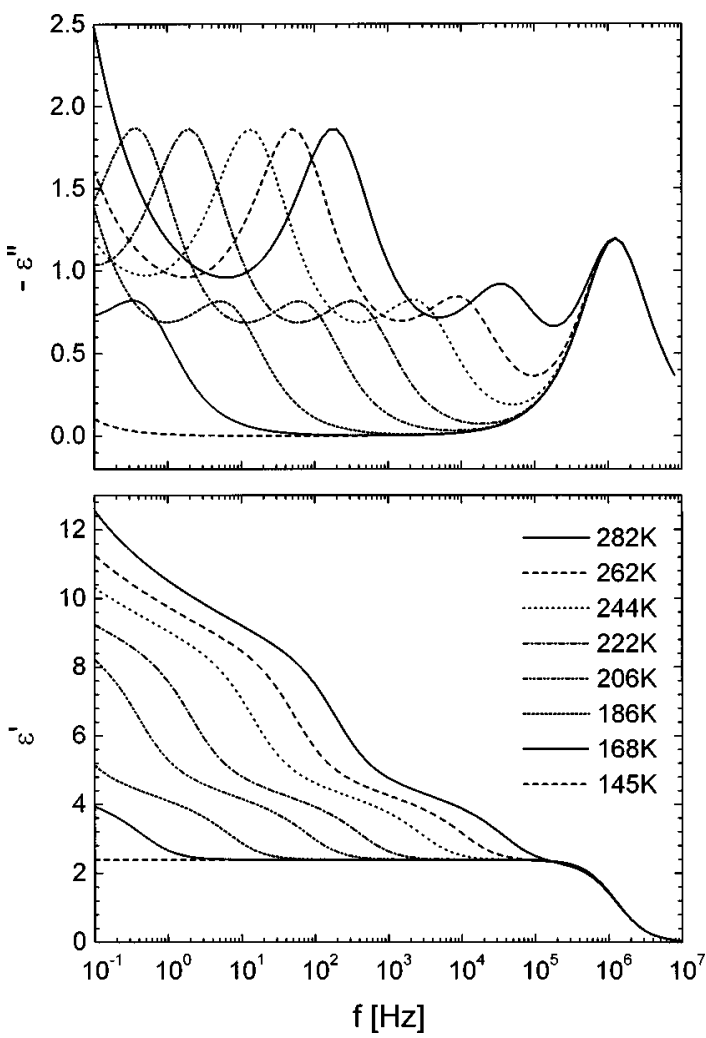

FIG. 6. Model calculation for the dielectric function $\hat{\varepsilon}$. The parameters used are described in the text. The essential features seen in Fig. 4, several maxima of $\varepsilon^{\prime \prime}$ (two of them $\mathrm{T}$ dependent) and a continuous rise of $\varepsilon^{\prime}$ are reproduced by the model. 
TABLE I. Parameters obtained by fitting Eq. (11) to the experimental data depicted in Fig. 4.

\begin{tabular}{lllllll}
\hline \hline Parameters & $186 \mathrm{~K}$ & $206 \mathrm{~K}$ & $222 \mathrm{~K}$ & $244 \mathrm{~K}$ & $262 \mathrm{~K}$ & $282 \mathrm{~K}$ \\
\hline$C_{l}[\mathrm{nF}]$ & 3.63 & 5.25 & 4.76 & 4.77 & 4.73 & 3.24 \\
$R_{l}[\Omega]$ & $2.08 \times 10^{9}$ & $2.99 \times 10^{7}$ & $5.37 \times 10^{6}$ & $8.51 \times 10^{5}$ & $1.63 \times 10^{5}$ & $6.2 \times 10^{4}$ \\
$\alpha_{l}$ & 0.36 & 0.50 & 0.55 & 0.56 & 0.62 & 0.57 \\
$C_{h}[\mathrm{nF}]$ & 2.58 & 2.43 & 2.20 & 2.28 & 2.63 & 2.46 \\
$R_{h}[\Omega]$ & $3.63 \times 10^{6}$ & $3.45 \times 10^{5}$ & $5.25 \times 10^{4}$ & $9.06 \times 10^{3}$ & $2.59 \times 10^{3}$ & $7.79 \times 10^{2}$ \\
$\alpha_{h}$ & 0.76 & 0.76 & 0.76 & 0.80 & 0.84 & 0.83 \\
$C_{\mathrm{gee}}[\mathrm{nF}]$ & 0.76 & 0.77 & 0.78 & 0.71 & 0.74 & 0.80 \\
$R_{0}[\Omega]$ & 73.24 & 70.81 & 68.13 & 69.15 & 65.00 & 69.80 \\
$\alpha_{0}$ & 1 & 1 & 1 & 0.98 & 0.91 & 0.88 \\
\hline \hline
\end{tabular}

demonstrate that the relative dielectric constant $\varepsilon_{r}$ determines the height and $R_{0}$ determines the right-hand slope of the first $\varepsilon^{\prime \prime}$ maximum from right. Therefore, if this relaxation process is resolved sufficiently one can unequivocally determine the values of $\varepsilon_{r}$ and $R_{0}$ from the height and the slope of this maximum. The second and third maxima of $\varepsilon^{\prime \prime}$ from right in Fig. 6 correspond to the processes in the higher and lower conductive bulk sublayer, respectively. The increase of $\varepsilon^{\prime \prime}$ at lowest frequencies depends on the width and hence the total resistance of the depletion layer. With decreasing temperature the second and third maxima of $\varepsilon^{\prime \prime}$ move to lower frequencies just as the experimental curves seen in Fig. 4. The height of the second maximum becomes a little lower with decreasing temperature, whereas the third maximum remains essentially unchanged. This is in contrast to the changes in the height of the corresponding experimental maxima in Fig. 4 but in the simplified model calculations the widths of the transition regions of the conductivity in the PPV layer are assumed to be independent of the temperature (only $s_{l}$ and $s_{h}$ are T dependent), which in reality may not be true. The conductivities of the sublayers determine the position of the maxima of $\varepsilon^{\prime \prime}$. But the height and the width of these maxima depend on the conductivity distribution. The spatial dependence of the conductivity of the PPV layer influences especially the real part $\varepsilon^{\prime}$. A model calculation for $\varepsilon^{\prime}$ with the same parameters as in Fig. 6, but now with an abrupt step function for $\sigma\left(\sigma=s_{j}\right.$ for $x \leqslant x_{l}$, $\sigma=2 s_{l}$ for $x_{l}<x \leqslant x_{h}$ and $\sigma=2 s_{h}$ for $\left.x_{h}<x \leqslant 1\right)$ instead of Eq. (10), shows that $\varepsilon^{\prime}$ curves exhibit clearly steps whereas one has in Fig. 6 an almost monotonous increase as in the experimental curves in Fig. 4. At room temperature $\varepsilon^{\prime}$ with the above abrupt step conductivity achieves a saturation value already at the frequency of about $10 \mathrm{~Hz}$, whereas $\varepsilon^{\prime}$ with $\sigma$ from Eq. (10) still increases. Due to the width of the transition region and the enormous resistance of the depletion layer the saturation value of $\varepsilon^{\prime}$ is in this case achieved only at very low frequencies not shown in Fig. 6.

In principle the theoretical expression (9) for the impedance should be fitted to the experimental data. However, the broadening of the maxima of $\varepsilon^{\prime \prime}$ and the smearing out of the steps of $\varepsilon^{\prime}$ caused by the spatial dependence of the conductivity can be simulated much simpler by using a Cole-Cole modification of the dielectric function Eq. (3) with the impedance Eq. (8) which can be written as

$$
\begin{aligned}
\hat{\varepsilon}= & \left\{\left(J \omega C_{\mathrm{geo}} R_{0}\right)^{\alpha_{0}}+\frac{C_{\mathrm{geo}}}{C_{j}} \frac{1}{1+\left(J \omega C_{j} R_{j}\right)^{-\alpha_{j}}}\right. \\
& \left.+\frac{C_{\mathrm{geo}}}{C_{l}} \frac{1}{1+\left(J \omega C_{l} R_{l}\right)^{-\alpha_{l}}}\right\}^{-1}, \\
& \left.+\frac{C_{\mathrm{geo}}}{C_{h}} \frac{1}{1+\left(J \omega C_{h} R_{h}\right)^{-\alpha_{h}}}\right\}^{-1}
\end{aligned}
$$

where $C_{\text {geo }}^{-1}=\Sigma_{i} C_{i}^{-1}$. Dielectric functions of this type are commonly used to describe dielectric relaxation processes in disordered media. The distribution parameters correspond to a distribution of relaxation times due to different local environments. However in our case, from the model calculations with the expression (9), it is clear that the Cole-Cole distribution parameters $\alpha_{i}$ are caused essentially by the inhomogeneity of the PPV conductivity.

The parameters obtained from the fit to the data of the sample of Fig. 4 are given in Table I. The fit parameters corresponding to the depletion layer are not reliable because $\varepsilon^{\prime}$ shows still no saturation in the low frequency region. Therefore they are not included in the table. From the fit we obtain an almost temperature independent value of about 70 $\Omega$ for the lead resistance $R_{0}$. The resistances of the plug-in connection with the measuring instrument and the coaxial lead resistance contribute only to a small part $(\approx 1-10 \Omega)$, thus the surface resistance of the ITO substrate gives the dominating contribution to $R_{0}$. With a sheet resistance of 20 $\Omega / \square$ an ITO stripe of $5 \mathrm{~mm}$ length and $5 \mathrm{~mm}$ width complies with the fitted lead resistance approximately. The fitted geometrical capacitance $C_{\text {geo }}$ gives a value of about 2.4 for the dielectric constant. $\varepsilon_{r}$ is here a little smaller than that determined from optical data $\varepsilon_{r} \approx 3-4 .^{31}$ The Cole-Cole parameter $\alpha_{0}$ in Eq. (11) is for all temperatures close to unity as expected. On the other hand $\alpha_{l}$ and $\alpha_{h}$ are much smaller than unity indicating a broader distribution. As explained in our model calculation this distribution arises from the continuous transitions between the differently conductive regions. Further, the capacitances of the low and high conductive regions are both almost temperature independent. This justifies the assumption of a constant $\varepsilon_{r}$ in our model calculation. As $C_{l}$ is slightly larger than $C_{h}$ the corresponding thickness of the lower conductive region should be smaller 


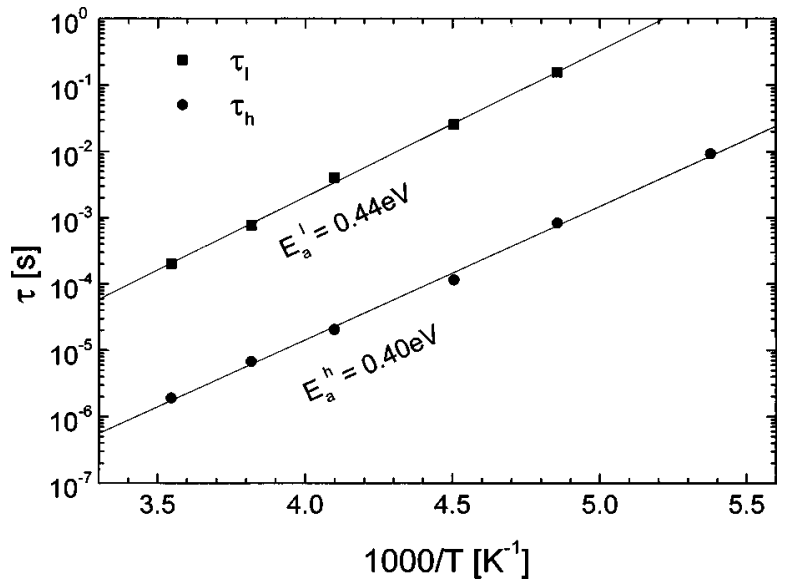

FIG. 7. Arrhenius plot of the relaxation times $\tau_{h}$ and $\tau_{l}$ obtained from the analysis of $\hat{\varepsilon}$ of the sample shown in Fig. 4 .

than that of the higher conductive region. Only the two resistivities $R_{l}$ and $R_{h}$ show a strong temperature dependence. It can be expressed best by the respective dielectric relaxation times $\tau_{i}=R_{i} C_{i}$. The dielectric relaxation time in a material is connected with its dc conductivity $\tau=\varepsilon_{0} \varepsilon_{r} / \sigma_{\mathrm{dc}}$. Because the dc conductivity of PPV is thermally activated we expect that the dielectric relaxation times of the two bulk processes $\tau_{i}$ show the same behavior. In Fig. 7 an Arrhenius plot of the bulk relaxation times is presented. The slope of the linear fit from $\ln \tau_{h}$ vs $1 / T$ results in an activation energy $E_{a}^{h}=0.4 \mathrm{eV} . \tau_{l}$ yields a little higher activation energy $E_{a}^{l}$ $=0.44 \mathrm{eV}\left(\tau_{l}\right.$ at $186 \mathrm{~K}$ is disregarded since this relaxation process is not observed in the available frequency region). These values are rather reasonable since our independent $\mathrm{dc}$ conductivity measurements of ITO/PPV/Al devices gave values for the activation energy in the same range. In Fig. 8 the temperature dependence of the conductivities for the high and low conducting regions calculated from $\tau_{h}$ and $\tau_{h}$ of the sample in Fig. 4 are compared with three other independent measurements: at first the same spectroscopic method is applied to a free-standing PPV film sandwiched between two

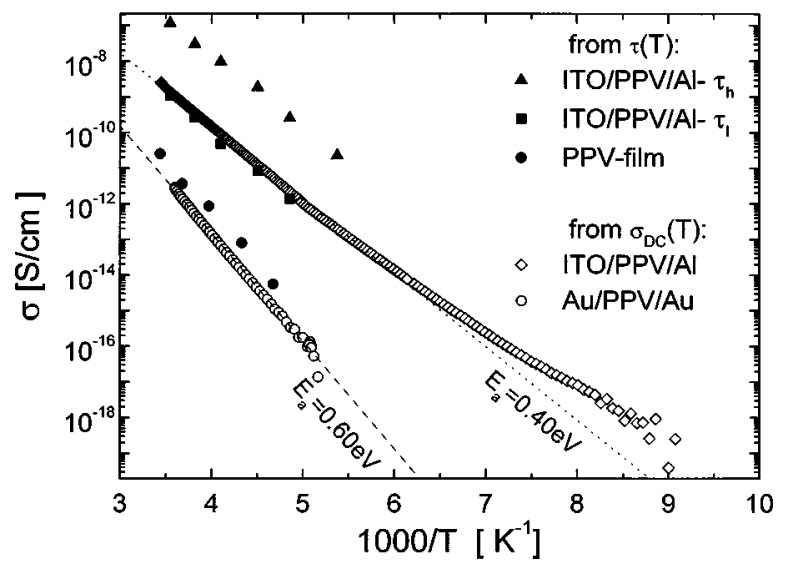

FIG. 8. Directly measured dc conductivities of an ITO/PPV/Al and a Au/ PPV/Au sample in comparison with the low and high conductivities calculated from the relaxation times which are obtained from fitting the dielectric function of the ITO/PPV/Al device (of Fig. 4) and the same for a freestanding PPV film (only one relaxation process is seen in this case). gold plates. In this case only one thermally activated process (activation energy about $0.6 \mathrm{eV}$ ) is obtained and the value of the conductivity is much smaller. Further for two different samples the results of direct dc measurements are given. One of them has also the ITO/PPV/Al configuration and is comparable with that of Fig. 4, but nevertheless these samples are different, the other one is a Au/PPV/Au device. Although it is tried to have constant synthesis and film fabrication conditions these conditions are determined by several parameters, whose number is unknown, examples are humidity, light influence during the synthesis process or differences in the conversion condition, and the metal evaporation. For this reason measured quantities show certain fluctuations from sample to sample. In view of the problems and uncertainties with the exact reproducibility of devices the agreement between the direct dc conductivity measurements and the data calculated from the dielectric relaxation times regarding the activation energy and also the absolute numbers of the conductivity (with the value of $\sigma_{l}$ calculated from $\tau_{l}$ for the ITO/PPV/Al device) is excellent. Figure 8 shows that the direct dc measurement on the diode gives practically (in view of actual device fabrication conditions) the same activation energy as obtained from both $\tau_{h}$ and $\tau_{l}$. At room temperature the conductivities of the higher and lower conductive bulk sublayers are about $10^{-7}$ and $10^{-9} \mathrm{~S} / \mathrm{cm}$, respectively. The value $4 \times 10^{-9} \mathrm{~S} / \mathrm{cm}$ of the dc conductivity, which measures the sum of both bulk sublayer resistances and hence the mean conductivity weighted by the widths of these sublayers, is only a little higher than $\sigma_{l}$. Both the free-standing film and the Au/PPV/Au sample have nearly the same and larger activation energy of about $0.6 \mathrm{eV}$ and their conductivity is at room temperature about three orders of magnitude lower than PPV on ITO. Both results indicate the role of the ITO in the unintentional doping process during the preparation of the ITO/PPV/Al diodes leading to the increase of the conductivity and a lowering of the activation energy to about $0.4 \mathrm{eV}$.

\section{Temperature dependence of the ionized acceptor dopant concentration in PPV}

In the preceeding subsections we have shown that detailed information on the internal structure of the PPV layer in the diodes can be obtained by appropriately analyzing the data from the dielectric impedance spectroscopy. More common for semiconductor/metal contacts is the measurement of the bias dependent capacitance of the depletion layer $C_{j}$. As discussed before, the junction capacitance should be consequently taken from the fits of the dielectric function. Its determination from bias sweeps at a fixed low frequency (as the common method) is in principle not sufficient in the case of the PPV diodes investigated here since even for the lowest accessible frequencies one has an increase of $\varepsilon$ " and no saturation of $\varepsilon^{\prime}$. Therefore, as the capacitance corresponds just to the latter its reliable determination is only possible in the low frequency saturation region of $\varepsilon^{\prime}$. As mentioned above, due to the enormous resistance of this layer (many orders of magnitude larger than in inorganic semiconductors like $\mathrm{Si}$ or $\mathrm{GaAs}$ ) and the continuous transition to the bulk, $\varepsilon^{\prime}$ of all the samples does not achieve saturation as demonstrated in Fig. 


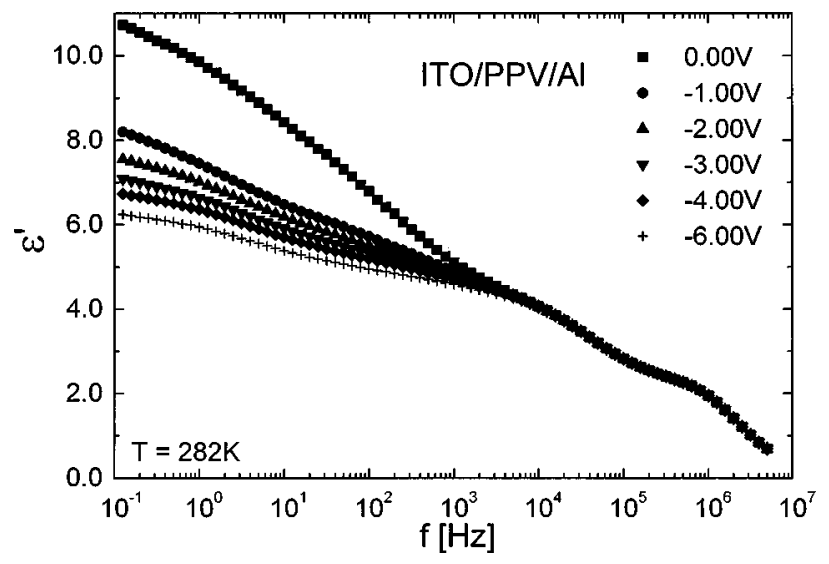

FIG. 9. Frequency dependence of $\varepsilon^{\prime}$ for the sample from Fig. 4 for different reverse bias values at $282 \mathrm{~K}$.

9. Thus, also the determination of this capacitance from the fit of the spectroscopic data is cumbersome. In addition, if a reserve bias is applied the width of the depletion layer becomes larger and the width of the lower conductive bulk sublayer smaller. Therefore the corresponding maximum in $\varepsilon^{\prime \prime}$ (i.e., the third maximum from right) becomes even less pronounced. When the depletion layer is wide enough, i.e., beyond some reserve bias voltage, this maximum disappears completely. This fact is seen as well in our model calculations as in the experimental data. This circumstance makes the fit procedure additionally more difficult. Therefore, in spite of its uncertainties we apply nevertheless the usual procedure to determine the junction capacitance. Under the assumption that at a reserve bias the depletion layer resistance is much larger than the serial bulk resistances, the junction capacitance $C_{j}$ is approximately the total measured low frequency (parallel) capacitance $C_{p}=-Y^{\prime \prime} / \omega$. For an abrupt junction the depletion layer capacitance of a Schottky contact is given by ${ }^{28}$

$$
C_{j}=A \sqrt{\frac{q \varepsilon_{0} \varepsilon_{r} N_{A}^{-}}{2\left(U_{D}-U\right)}},
$$

where $N_{A}^{-}$is the ionized acceptor concentration and $U_{D}$ the diffusion voltage. Thus a linear plot of $1 / C_{j}^{2}$ versus bias voltage should give a straight line with a slope proportional to $1 / N_{A}^{-}$. The depletion layer width $w$ is then given by

$$
w=\sqrt{\frac{2 \varepsilon_{0} \varepsilon_{r}\left(U_{D}-U\right)}{q N_{A}^{-}} .}
$$

We have to mention that such a linear dependence has not been obtained in all samples. An example for a nonlinear behavior is shown in Fig. 10 for an ITO/PPV/Ca sample ( $d$ $=500 \mathrm{~nm})$ at different temperatures. These experimental data are measured at $10 \mathrm{~Hz}$. From reserve bias $U=-5 \mathrm{~V}$ to about $U=-1 \mathrm{~V}$ the plot of $1 / C_{j}^{2}$ vs $U$ is linear, however, between $U=-1$ and $U=2 \mathrm{~V}$ deviations from this behavior are observed. The absolute values of $N_{A}^{-}$determined by Eq. (12) between -5 and $-1 \mathrm{~V}$ have a considerable error, but the tendency of a decrease of $N_{A}^{-}$with decreasing temperature is clearly seen. Such deviations from the linear $1 / C_{j}^{2}$ versus bias behavior can be caused by two reasons. The first reason

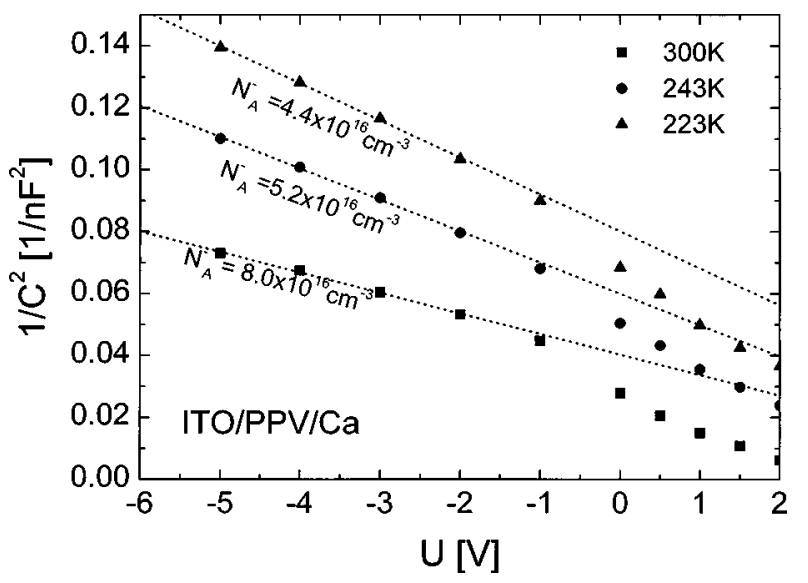

FIG. 10. The reciprocal squared capacitance as bias-dependent function for an ITO/PPV/Ca sample measured at $10 \mathrm{~Hz}$ at different temperatures. The acceptor dopant concentration is obtained accordind to Eq. (12) from linear fits between -5 and $-1 \mathrm{~V}$. Above $-1 \mathrm{~V}$ deviations from the linear behavior are observed.

is found in the inhomogeneity of the acceptor depth profile in the bulk layer as discussed in the preceding section. In this case, it is in principle possible to obtain the doping depth profile from the derivative of $1 / C_{j}^{2}$ [from Eq. (12)] with respect to the voltage $U,{ }^{28}$ provided that the assumption of an abrupt junction is correct. Given typical values of the diffusion voltage $U_{D} \approx 1.5 \mathrm{~V}$ and the depletion layer width at zero bias $w \approx 100 \mathrm{~nm}$, a variation of the bias between -5 and $+1 \mathrm{~V}$ would allow to probe the ionized acceptor density in a depth between about 60 and $210 \mathrm{~nm}$ to the metal electrode. This distance is less than half of the total polymer thickness for the devices investigated here. Thus, the visibility of the inhomogeneous doping profile in capacitance-voltage spectroscopy depends on the total polymer thickness and may not be seen in all devices.

On the other hand, systematic studies of polymeric Schottky diodes by Taylor et al. ${ }^{32}$ show that deviations from a linear $1 / C_{j}^{2}$ vs $U$ behavior can also result from two (or more) sets of acceptors (traps) at different energies in the energy gap of the polymer. A change of slope in the $1 / C_{j}^{2}$ vs $U$ plot occurs when the applied bias is of such a magnitude that the Fermi energy crosses the energy of a localized level. This is also a possible explanation for the investigated PPV devices, since our recent measurements using thermally stimulated currents indicate the presence of several distinct gap states with energies in the range $0.05-0.2$ and $0.6-1$ $\mathrm{eV} .{ }^{20}$ While the former ones could be clearly assigned to the doping by the ITO substrate, the latter ones are due to the influence of the environment (oxygen) and are very likely to vary from sample to sample. Thus, unless further investigations of this issue are performed, it is not possible to decide which one of the above explanations is true for the observed nonlinear $1 / C_{j}^{2}$ vs $U$ characteristics.

Therefore, for the following discussion of the temperature dependence of the ionized acceptor concentration, we have chosen samples, which showed a linear $1 / C_{j}^{2}$ vs $U$ behavior. Figure 11 shows the reciprocal of the squared junction capacitance of an ITO/PPV/Al sample $(d=600 \mathrm{~nm})$ measured at $0.16 \mathrm{~Hz}$ as a bias-dependent function for various 


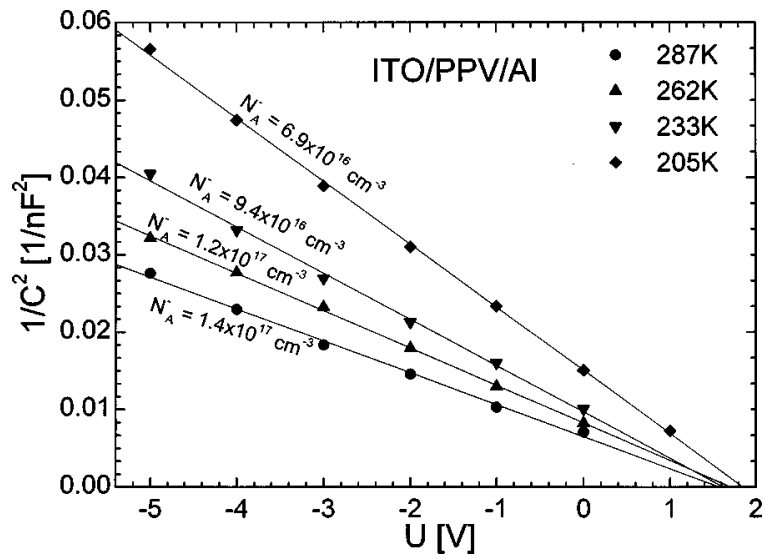

FIG. 11. The reciprocal squared capacitance as bias-dependent function for an ITO/PPV/Al sample $\left(d=600 \mathrm{~nm}, A=25 \mathrm{~mm}^{2}\right)$ measured at $0.16 \mathrm{~Hz}$ at different temperatures. The acceptor dopant concentration is obtained according to Eq. (12) from linear fits between -5 and $1 \mathrm{~V}$.

temperatures. Here the linear behavior of $1 / C_{j}^{2}$ versus bias voltage $(U \leqslant 1 \mathrm{~V})$ in reserve direction is clearly seen. This means that the concept of a simple Schottky junction at the interface can be applied to this device. All linear fits in Fig. 11 give a value of about $1.5-1.7 \mathrm{~V}$ for the diffusion voltage, which is comparable with our earlier results. ${ }^{12}$ As mentioned above, $C_{j}$ is here the capacitance at a fixed low frequency. However, at and below $0.16 \mathrm{~Hz} \varepsilon^{\prime}$ still increases (see for example Figs. 3 and 9). Therefore, the error of the absolute value of the doping concentration $N_{A}^{-}$determined from the slope of the linear plot is considerable (a factor 2 is possible). Nevertheless, the tendency of the temperature dependence of $N_{A}^{-}$from Fig. 11 is significant: With decreasing temperature (from 287 to $205 \mathrm{~K}$ ) $N_{A}^{-}$decreases from $1.4 \times 10^{17}$ to 6.9 $\times 10^{16} \mathrm{~cm}^{-3}$, whereas $U_{D}$ obtained from the intercept remains constant at about $1.6 \mathrm{~V}$. The corresponding depletion layer width at zero bias increases from about $55 \mathrm{~nm}$ at room temperature to about $80 \mathrm{~nm}$ at $205 \mathrm{~K}$.

From earlier work a temperature dependence of the depletion layer capacitance measured at $222 \mathrm{~Hz}$ is known. ${ }^{12,30}$ In this work, at room temperature $C_{j}$ achieves almost saturation. Between 260 and $220 \mathrm{~K}$ it drops down abruptly to the geometrical capacitance. The usual interpretation for this decrease of the capacitance is the freeze out of mobile charge carriers (ionized acceptor concentration $N_{A}^{-}$). Consequently the width of the depletion layer increases and hence $C_{j}$ decreases upon cooling. The saturation of $C_{j}$ at room temperature corresponds consequently to the complete acceptor ionization. Our measurements show that $C_{j}$ not only depends on temperature but also on the measurement frequency. From Fig. 9 it is seen that for a frequency higher than $1 \mathrm{kHz} \varepsilon^{\prime}$ and thus $C_{j}$ is practically bias independent already at room temperature and consequently also at lower temperatures. At a frequency of $100 \mathrm{~Hz} C_{j}$ will be bias dependent at room temperature, however with decreasing temperature the steps in $\varepsilon^{\prime}$ move to lower frequencies and $C_{j}$ will soon drop to the geometrical capacitance $C_{\text {geo }}$. This is a direct consequence of the dielectric relaxation time which decreases during cooling. If the measurement frequency is larger than $1 / \tau$ the mobile charge carriers cannot respond to

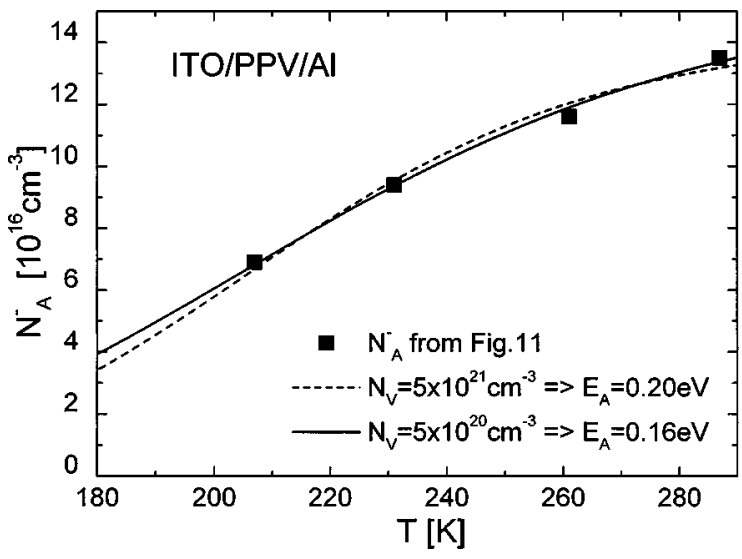

FIG. 12. Temperature dependence of the ionized acceptor concentration for the sample in Fig. 11 and fits with Eq. (14) using two different densities of valence band states.

the applied electric field and hence the capacitance decreases, too. Therefore, the abrupt decrease of $C_{j}$ in such a narrow $\mathrm{T}$ interval is caused not only by freezing out mobile charge carriers but also by the fact that the measurement frequency, though small, is still large compared to $1 / \tau$ for this low conductive material. Thus for a temperature dependent determination of $C_{j}$ it is necessary to measure the bias dependence at the lowest possible frequencies. In our case the lower frequency limit was $0.1 \mathrm{~Hz}$.

One can obtain the temperature dependence of the ionized acceptor concentration as in conventional semiconductors from

$N_{A}^{-}=-\frac{1}{2} N_{V} \exp \left(-\frac{E_{A}}{k_{B} T}\right)\left[1-\sqrt{1+4 \frac{N_{A}}{N_{V}} \exp \left(\frac{E_{A}}{k_{B} T}\right)}\right.$,

where $E_{A}$ is the acceptor ionization energy, $N_{V}$ the effective density of states in the valence band, and $N_{A}$ the density of electrically active acceptors. For the sample shown in Fig. 11 we have only four values for the density of ionized acceptors $N_{A}^{-}(T)$ presented in Fig. 12. It should be noted that $C_{j}$ of all our samples does not saturate at room temperature. This means that the condition of the complete acceptor ionization at room temperature is not sufficiently fulfilled. Nevertheless, assuming $N_{A}^{-} \approx 90 \% N_{A}$ at room temperature one can try to fit Eq. (14) to the calculated data of $N_{A}^{-}(T)$ shown in Fig. 12. With $N_{V}=5 \times 10^{21}$ and $5 \times 10^{20} \mathrm{~cm}^{-333,34}$ this fit yields $E_{A}=0.2$ and $0.16 \mathrm{eV}$, respectively. Such low values are in contrast to the activation energy obtained from direct dc conductivity measurements and the relaxation times from impedance spectroscopy $\left(E_{a}=0.4 \mathrm{eV}\right)$. However, the data cannot be fitted using $E_{A}=E_{a}=0.4 \mathrm{eV}$ and $N_{V}=10^{21} \mathrm{~cm}^{-3}$ : for these parameters $N_{A}^{-}$is only $2 \%$ of $N_{A}$ at room temperature. From our data one has to conclude that the characteristic energies responsible for the transport in the PPV bulk (with $E_{a}=0.4 \mathrm{eV}$ for ITO substrates) and the temperature dependent behavior of the depletion layer (leading to an acceptor energy $E_{A}=0.16-0.2 \mathrm{eV}$ ) are significantly different. So far in dc conductivity and mobility measurements by our group and others activation energies between 0.4 and 0.9 $\mathrm{eV}^{19,26,29,35}$ (depending on the substrate and environmental 
conditions) have been found with no indication for acceptor states with energies lower than $0.2 \mathrm{eV}$. On the other hand, our recent TSC measurements ${ }^{20}$ yield two sets of traps levels with energies of $0.05-0.2$ and $0.6-1 \mathrm{eV}$, respectively. While the deep traps can be assigned to the influence of air and their density can be varied by applying vacuum to the devices, the shallow traps could be identified as due to doping by the ITO substrate. Comparable shallow trap states have been found from an analysis of current-voltage characteristics using space charge limited transport with an exponential trap distribution on ITO/PPV/Al devices with Cambridge PPV. ${ }^{11}$ From these observations one has to conclude that the electrical properties of PPV based devices are determined by at least two different energetic states in the gap of the material. The observed activation energies of macroscopic quantities can then be a combination of these levels, the value of which probably depends on the relative concentration of the different gap states. The clarification of the microscopic nature of these trap states and their role in charge transport needs further investigation.

\section{CONCLUSIONS}

In this article broadband dielectric spectroscopy in the frequency region from $0.1 \mathrm{~Hz}$ to $10 \mathrm{MHz}$ and in a temperature interval from 300 to $145 \mathrm{~K}$ has been applied to forward and reverse biased ITO/PPV/Al diodes. It has been demonstrated that this method considerably extends the possibilities of the electrical characterization of such systems. Most important, combining the method with information on the preparation and with an appropriate theoretical description one can obtain not only information on the rectifying PPVmetal contact but also on the bulk of the PPV film, especially on the temperature dependence and the spatial variation of the conductivity in the film. It has been found that the usual representation of the impedance as a Cole-Cole plot in the complex impedance plane does not reveal the complete frequency dependent behavior. Instead, the complex dielectric function is more appropriate to characterize these devices. This quantity displays two relaxation times, which can be assigned to two regions of the PPV bulk with different conductivity. Their temperature dependence correlates well with the directly measured dc conductivity of PPV.

Information about the ionized acceptor concentration in PPV has been obtained from the bias dependence of the depletion layer capacitance at the PPV/Al interface. The temperature dependence of this quantity yields an acceptor depth between 0.16 and $0.2 \mathrm{eV}$, which is significantly lower than the activation energies of the bulk relaxation processes with $0.4-0.6 \mathrm{eV}$. This indicates that the transport properties of PPV are influenced by at least two sets of gap states with different energies.

\section{ACKNOWLEDGMENTS}

We thank M. Meier and S. Karg for helpful discussions and J. Gmeiner for polymer synthesis. Financial support from the Bayerische Forschungsstiftung (FOROPTO) and from the Deutsche Forschungsgemeinschaft is gratefully acknowledged.

1 T. A. Skotheim, Handbook of Conducting Polymers (Marcel Dekker, New York, 1986).

${ }^{2}$ W. R. Salaneck, I. Lundström and B. Rånby, Conjugated Polymers and Related Materials (Oxford University Press, Oxford, 1993).

${ }^{3}$ N. C. Greenham and R. H. Friend, in Solid State Physics, edited by H. Ehrenreich and F. Spaepen (Academic, Boston, 1995), Vol. 49.

${ }^{4}$ J. H. Burroughes, D. D. C. Bradley, A. R. Brown, R. N. Mackay, R. H. Friend, P. L. Burn, and A. B. Holmes, Nature (London) 347, 539 (1990).

${ }^{5}$ D. Braun and A. J. Heeger, Appl. Phys. Lett. 58, 1982 (1991).

${ }^{6}$ D. D. C. Bradley, Synth. Met. 54, 401 (1993).

${ }^{7}$ W. Rieß, in Organic Electroluminescent Materials and Devices, edited by S. Miyata and H. Nalwa (Gordon \& Breach Science, London, 1996).

${ }^{8}$ N. Tesler, N. T. Harrison, and R. H. Friend, Adv. Mater.10, 64 (1998).

${ }^{9}$ I. D. Parker, J. Appl. Phys. 75, 1656 (1994).

${ }^{10}$ R. N. Marks, D. D. C. Bradley, R. W. Jackson, P. L. Burn, and A. B. Holmes, Synth. Met. 55, 4128 (1993).

${ }^{11}$ A. J. Campbell, D. D. C. Bradley, and D. G. Lidzey, J. Appl. Phys. 82, 6326 (1997).

${ }^{12}$ M. Meier, S. Karg, and W. Rieß, J. Appl. Phys. 82, 1961 (1997).

${ }^{13}$ S. Karg, M. Meier, and W. Rieß, J. Appl. Phys. 82, 1951 (1997).

${ }^{14}$ M. Herold, J. Gmeiner, C. Drummer, and M. Schwoerer, J. Mater. Sci. 32, 5709 (1997).

${ }^{15}$ G. Sauer, M. Kilo, M. Hund, A. Wokaun, S. Karg, M. Meier, W. Rieß, M. Schwoerer, H. Suzuki, J. Simmerer, H. Meyer, and D. Haarer, Fresenius J. Analyt. Chem. 353, 642 (1995).

${ }^{16}$ W. Brütting, M. Meier, M. Herold, S. Karg, and M. Schwoerer, Chem. Phys. Lett. 227, 243 (1998).

${ }^{17}$ M. G. Harrison, J. Grüner, G. C. W. Spencer, Synth. Met. 76, 71 (1996).

${ }^{18} \mathrm{H}$. Antoniadis, B. R. Hsieh, M. A. Abkowitz, S. A. Jenekhe, and M. Stolka, Synth. Met. 62, 265 (1994).

${ }^{19}$ T. P. Nguyen and V. H. Tran, Mater. Sci. Eng., B 31, 255 (1995).

${ }^{20}$ M. Meier, S. Karg, K. Zuleeg, W. Brütting, and M. Schwoerer, J. Appl. Phys. (submitted).

${ }^{21}$ J. R. MacDonald, Impedance Spectroscopy (Wiley, New York, 1987).

${ }^{22} \mathrm{P}$. Blood and J. W. Orton, in The Electrical Characterization of Semiconductors: Majority Carriers and Electron States Techniques of Physics (Academic, London, 1992).

${ }^{23}$ C. J. F. Böttcher and P. Bordewijk, in Theory of Electric Polarization (Elsevier, Amsterdam, 1978), Vols. 1 and 2.

${ }^{24}$ A. K. Johnscher, Dielectric Relaxation in Solids (Chelsea Dielectrics, London, 1983).

${ }^{25}$ R. M. Fleming and R. J. Cava, in Low Dimensional Electronic Properties of Molybdenum Bronzes and Oxides, edited by C. Schlenker (Kluwer, Dordrecht, 1989).

${ }^{26}$ J. Gmeiner, S. Karg, M. Meier, W. Rieß, P. Strohriegl, and M. Schwoerer, Acta Polym. 44, 201 (1993).

${ }^{27}$ M. Herold, J. Gmeiner, and M. Schwoerer, Acta Polym. 47, 436 (1996).

${ }^{28}$ S. M. Sze, Physics of Semiconductor Devices (Wiley, New York, 1981).

${ }^{29}$ G. Paasch, W. Rieß, S. Karg, M. Meier, and M. Schwoerer, Synth. Met. 67, 177 (1994).

${ }^{30}$ S. Karg, Dissertation, Universität Bayreuth (1994).

${ }^{31}$ F. Bauer, Dissertation, Universität Bayreuth (1995).

${ }^{32}$ D. M. Taylor and H. L. Gomes, J. Phys. D: Appl. Phys. 28, 2554 (1995).

${ }^{33}$ P. S. Davids, A. Saxena, and D. L. Smith, J. Appl. Phys. 78, 4244 (1985).

${ }^{34}$ G. Paasch, P. H. Nguyen, and S.-L. Drechsler, Synth. Met. (submitted).

${ }^{35}$ E. Lebedev, Th. Dittrich, V. Petrova-Koch, S. Karg, W. Brütting, Appl. Phys. Lett. 71, 2686 (1997). 